\title{
Macroscopic Traffic Dynamics in Urban Networks during Incidents
}

\author{
Sasan Amini (Corresponding Author) \\ Chair of Traffic Engineering and Control \\ Technical University of Munich, Arcisstr. 21, 80333 Munich, Germany \\ ORCID: 0000-0002-1675-4945 \\ Email: sasan.amini@tum.de \\ Gabriel Tilg \\ Chair of Traffic Engineering and Control \\ Technical University of Munich, Arcisstr. 21, 80333 Munich, Germany \\ ORCID: 0000-0001-9167-0680 \\ Email: gabriel.tilg@tum.de

\section{Fritz Busch} \\ Chair of Traffic Engineering and Control \\ Technical University of Munich, Arcisstr. 21, 80333 Munich, Germany \\ ORCID: 0000-0002-2194-548X \\ Email: fritz.busch@tum.de
}

Word count: 5325 words +2 table(s) $\times 250=5825$ words

Paper submitted for presentation at the $99^{\text {th }}$ Annual Meeting Transportation Research Board, Washington D.C., January 2020 
1 ABSTRACT

2 The degradation of road network performance due to incidents is a major concern to traffic operators. The development of urban traffic incident management systems requires a comprehensive understanding of traffic dynamics during incidents. Recently, the concept of the macroscopic fundamental diagram (MFD) contributed to such an understanding and has been used in a wide range of applications. However, the MFD is merely reproducible under recurring traffic patterns. Motivated by a few studies which argue the existence of the MFD with a clockwise hysteresis loop during incidents, we tackle this limitation of the MFD and propose a framework to study the characteristics of the MFD under non-recurring congestion. More specifically, we introduce a criticality 


\section{INTRODUCTION}

With the growth of traffic volumes in urban networks, traffic congestion has become a major concern for both citizens and city authorities. It increases travel time, air pollution and reduces economic productivity, safety, and quality of life. Generally, there are two types of congestion: (i) recurring congestion that occurs regularly when demand exceeds the road capacity, for example, every day during peak hours, and (ii) non-recurring congestion which is caused by incidents or planned special events which either temporarily reduce supply or suddenly increase demand. It is critical to distinguish these two as their required countermeasures are inherently different. The degradation of road network performance due to non-recurring congestion, hereafter called incidents, is a much bigger concern to traffic operators.

Incidents are generally defined as situations where the observed traffic state significantly deviates from its prediction (1). Most of the incident detection methods base their algorithm on this logic and hence, all of them, in some form or another, are comparative algorithms (2). Incident detection methods can be divided into two very broad groups. First, parametric statistical methods such as the class of time series analysis or state-space models, as well as non-parametric models which include the family of machine learning techniques e.g. neural networks or support vector machines. Second, methods that are based on the traffic flow theory such as e.g. McMaster Algorithm (3).

The latter group mainly consists of methods that are based on the well-known fundamental diagram of traffic flow. The advantage of these models in comparison to the previous group is that they use explanatory variables that characterize traffic dynamics. However, the notation of the fundamental diagram is limited to uninterrupted facilities. Consequently, these incident detection algorithms e.g. $(4,5)$ are mainly confined to freeway segments. Nonetheless, a limited number of studies have been conducted to develop similar incident detection in urban arterials. Bell and Thancanamootoo (6) were the first to use flow and occupancy data from loop detectors of adaptive traffic signal control system SCOOT to detect incidents. Sethi et al. (7) have developed two distinct algorithms for fixed loop detectors and probe vehicles that can be applied on single links. However, these methods are extremely biased and depend on the positioning of the detectors, probe penetration rate, the time resolution of data and fail to detect the congestion where no detector is available e.g. if traffic diverges on the upstream links.

The group of incident detection methods mentioned first handles the problem of incident detection mostly as temporal outlier detection in single-profile sensor data using signal processing techniques. These methods have emerged from other fields such as wireless sensors or internet traffic analysis and vary from simple boxplot methods to support vector machines (8). However, these methods face two challenges. First, they often require labeled data as ground truth or rely on a prioiri data distributions. In reality, however, such information is rarely available for urban traffic. Data are often noisy and might be recorded by malfunctioning sensors. Second, urban road networks as a physical system have much more complicated dynamics in comparison to wireless sensor networks. Nevertheless, various researchers have applied these methods on urban networks to detect incidents.

For instance, Anbaruglu et al. (9) proposed a spatiotemporal clustering approach to detect non-recurring congestion in London using travel time data from automated number plate recognition (ANPR) cameras. They employ a global constant factor to decide whether the travel time of a link is exceeding the normal level. Such an assumption will not represent the heterogeneity of urban traffic dynamics. The authors extended their work in a follow-up (10) and relaxed the as- 
sumption of a constant congestion factor by proposing a space-time scan statistics which annotates congestion as non-recurring if the difference between current and historical travel times is statistically significant. In another study, Zhang et al. (11) use dictionary-based compression theory to identify the features of both spatial and temporal patterns by analyzing multi-dimensional trafficrelated data. We briefly review some of these studies in Section 2 to present a comprehensive oversight.

Both of these two groups of methods require large historical data sets and extensive sensor networks, which are not available in many cities. Moreover, they are mainly applied for incident detection. They do not evaluate the network performance, and thus, cannot be used to develop proper traffic management strategies. In this paper we try to fill this gap by characterizing the impact of incidents on the performance of urban networks using recent findings in traffic flow theory i.e. the concept of the macroscopic fundamental diagram (MFD). Recently, several studies suggesting that instability in network caused by major disturbances imposes clockwise hysteresis loops in the MFD were published. The question is if the disturbances based on their impact on the shape of MFD can be clustered and use such clusters to detect similar exogenous instabilities. The contribution of the paper is twofold. First, we propose a framework that can not only be used for incident detection but also for development of efficient traffic management strategies to alleviate incidents impacts. Secondly, we study the properties and characteristics of MFD during non-stationary states which has received very little attention.

The remainder of this paper is structured as follows. The theoretical background and properties of the MFD is provided in Section 2. Then we introduce our approach for characterization of incidents in urban networks in Section 3. In Section 4 we present a microscopic traffic simulation case study to evaluate the efficiency of the methodology. The results of this case study are discussed in Section 5. We provide conclusions and directions for future work in Section 6.

\section{THEORETICAL BACKGROUND}

\section{Macroscopic Fundamental Diagram}

The original idea of a macroscopic relationship between network outflow and accumulation was first introduced by Godfrey (12). Several researchers postulated a similar relationship $(13,14,14)$ and Mahmassani (15) tested it in a simulation experiment. But it was only after the work of Daganzo and Geroliminis $(16,17)$ that the MFD gained attention. Inspired by their findings on data from Yokohama, many other researchers have observed a similar relationship in empirical data from several cities such as Toulouse, France (18), Brisbane, Australia (19), Zurich, Switzerland (20), Lucerne, Switzerland, and London in the United Kingdom (21), as well as in traffic simulations $(22,23,24)$. The MFD is arguably network-specific and a function of network topology, signal control settings and route choice $(22,23)$. This has made it a valuable tool to develop a wide range of traffic control applications such as routing strategies $(25,26,27)$, perimeter traffic control $(28,29)$, on-street parking $(30,31)$ and congestion charging $(21)$. While influential factors on the shape of MFD has been well studied under steady states, empirically derived MFDs exhibit multivaluedness and hysteresis loops which need to be further investigated.

\section{Bifurcation and Hysteresis Loops in the MFD}

Bifurcation or hysteresis loops are certain types of multivaluedness in the MFD where two groups of flow values for a given range of density are observed.

The first empirical hysteresis loop was reported in Toulouse (18) where on a particular day 
due to a social movement of truck drivers between 8 and 10 in the morning the speed on the city's rind road drops and causes drivers to search for alternatives. Motivated by this observation, Gayah and Daganzo (32) showed that even in a perfectly symmetric network with uniform demand, a hysteresis loop forms during network unloading. They identify four types of MFD paths i.e. the single-path, the clockwise loop, the counterclockwise loop, and the figure-Eight. They conjecture that during recovery, when more trips are ending than starting, the network has a tendency towards uneven congestion distribution which results in a clockwise hysteresis loop. Saberi et al. $(33,34)$ have observed a similar phenomenon in a freeway network. Others have studied the potential reasons for hysteresis-like patterns in the MFD $(25,35,36)$. Sim et al. (36) propose four possible reasons for bifurcation in empirical MFD: network heterogeneity, trip completion rate, detouring pattern, and commuting trips. Geroliminis and Sun (37) have conducted a thorough analysis of network heterogeneity and argue that hysteresis is a result of multiple congestion pockets of different size which are not necessarily interconnected. Since arterial networks offer a higher degree of redundancy, drivers adaptively change their routes to identify less congested links. Consequently, the distribution of congestion is more homogeneous whereas freeway networks are not redundant and therefore, congestion propagates unevenly. This assumption may not hold during non-recurring congestion especially when drivers cannot make perfect route choices. For example, in an empirical study in Brisbane, Australia (19) an abnormal scatter and capacity drop is observed because of an accident on a major arterial resulting in a road closure during morning peak hours. The authors concluded that if network partitioning is performed, MFD can be used to detect incidents.

The literature in this particular line of research i.e. the shape of MFD during non-recurring congestion is very limited. For instance, Zockaie et al. (38) study the shape of the MFD during the evacuation of a large-scale city. They conclude that adaptive drivers increase fluctuations in the MFD but reduce hysteresis and improve capacity. In a more recent study, Kim et al. (39) use the concept of MFD to develop a novel link criticality index. They remove a link from a network and assume the duration of the closure is short enough that neither a new user equilibrium is formed nor OD demand changes significantly. The authors focus only on network loading and incrementally increase a random demand function until the network reaches gridlock. The obtained MFD shows slightly lower flow for the same density of the normal operation, lower capacity but the same value near jam density. Based on this performance loss, the criticality of each link is evaluated. Lastly, Horiguchi et al. (40) propose a mesh-wised network monitoring framework to detect anomalous congestion. They introduce a fluidity indicator which is based on the distance of the obtained MFD point from the origin $(0,0)$ and a singularity indicator which shows the probability to observe a point at a certain time of day. In consideration of these studies, the question arises if similar disruptions in dense urban networks cause comparable deviations in the shape of the MFD.

\section{METHODOLOGY}

In this section, we propose the methodology for the analysis and characterization of traffic flow dynamics during incidents in urban networks. It is based on the concept of the MFD and is composed of two main parts. The first part deals with the criticality of each link in a network by introducing a criticality score (CS) that reflects the impact of closing each road on the performance of the network. The second part of the methodology quantifies the properties of MFDs using a set of explanatory variables.

Then, for a given network with predefined OD demand, we postulate that closing links with similar CS results in a comparable change in the shape MFD. In other words, the characteristics 
of the scatter and hysteresis loop imposed on the flow-density plane of the MFD by an incident can be related to a set of features explaining how critical that link is for the network. Quantifying the impact of incidents and linking them to the characteristics of the network allows us to detect incidents under incomplete sensor coverage and improve network performance by implementing efficient traffic management measures. Please note that the main assumption is that demand is recurrent. This implies that any deviation from the expected MFD curve is caused by exogenous disruptions.

\section{Criticality Score}

First, we define our criticality score (CS), which is similar to the definition of betweenness centrality in graph theory, generalized for directed graphs by White and Borgatti (41). In network science, edge Betweenness centrality is often applied to indicate how critical an edge is to a given graph. It is defined as the fraction of shortest paths between all pairs of nodes passing through an edge. A higher value of a betweenness centrality represents a bridge-like connector between two parts of a graph. The removal of such a connector may affect the shortest path between many pairs of nodes. In transportation networks, shortest paths are usually defined based on generalized cost including distance, travel time, comfort etc. which are time-varying and depend mostly on the level of congestion. Calculating such a dynamic cost function requires a comprehensive understanding of the network, which is usually missing. In addition to the length of the shortest path, it is important to evaluate the number of existing alternatives for the shortest path. This feature is usually referred to as redundancy and considers the number of alternative paths for a given OD pair. In the case of a road closure, redundancy plays a vital role in compensating the impacts of closure through an even distribution of congestion. Hence, it is a critical feature of the network. Therefore, instead of betweenness centrality, we propose a simpler alternative approach focusing only on redundancy for homogeneous networks i.e. networks with links with identical fundamental diagram, equal length and traffic signal configuration as follows.

Let $\mathscr{G}(V, E)$ be a directed graph representing the road network where $V$ and $E$ are the sets of nodes and edges, respectively. We denote the adjacency matrix of the network by $\mathscr{A}$ and calculate the number of all shortest paths between each pair of nodes. Using simple graph theory, one can prove that the number of paths with length $k$ between node $\mathrm{i}$ and $\mathrm{j}$ is the $(\mathscr{A})_{i j}^{k}$ where $\mathscr{A}^{k}$ is $k$ th power of Matrix $\mathscr{A}$. In other words, the number of paths with length $\mathrm{k}$ from node $\mathrm{i}$ to node $\mathrm{j}$ is equal to the element $a_{i, j}^{k}$ of matrix $\mathscr{A}^{k}$. Thus, the total number of all possible paths for network $\mathscr{A}$ from length of 1 to maximum possible length $k_{\max }$ is:

$$
N_{p}^{A}=\sum_{(i, j)} \sum_{k=1}^{k_{\max }} a_{i j}^{k} \text { for }(i, j) \in V \times V
$$

We define the CS of a link by the reduction in the total number of paths once we close that link $C S_{l}=N_{P}^{A}-N_{P}^{A^{l}} . A^{l}$ represents the adjacency matrix of the network after removal of link $l$. Higher values of CS indicate that more drivers must be rerouted to reach their destination and some links become oversaturated due to lack of alternative routes between the first-order adjacency of the closed link. Such an assumption implies that drivers learn about the closure just before they reach the closed road which is realistic in case of unexpected incidents. In our use case, this is particularly important because as suggested in the literature (33) adaptive drivers have a significant influence on the shape of hysteresis loops and network capacity. Please note that this is only true 
1 for homogeneous networks with an existing MFD.

\section{Characterization of the MFD Hysteresis Loop}

In order to categorize the incidents, we must quantify their impacts on the MFD. We use a set of explanatory variables to measure the differences between two MFDs. Saberi and Mahmassani (34) have characterized the hysteresis loop by its shape. They describe a loop by its height $\Delta q$, width $\Delta k$, and the area covered by this rectangular $S_{H}=\Delta q \times \Delta k$. However, these variables are not enough to identify a hysteresis loop uniquely. For example, as it is shown in Figure 1, two hysteresis loops can have an identical area but one (MFD 2 in Figure 1) shows higher average speed. Thus, we take into account the average speed of traffic states related to the hysteresis loop. Furthermore, incidents cause a capacity loss in the MFD which should be measured as well denoted by $q_{n i}$. Additionally, it is important to consider the number of observations that form the hysteresis loop, as an indicator for the duration of instability in the MFD. Table 1 summarizes the explanatory variables to describe the MFD. It is important to note that we avoid replacing density with occupancy to reduce potential error sources while converting occupancy to density. Only for the calculation of average speeds, we assume an average vehicle length of $5 \mathrm{~m}$ and a detector length of $2 \mathrm{~m}$.

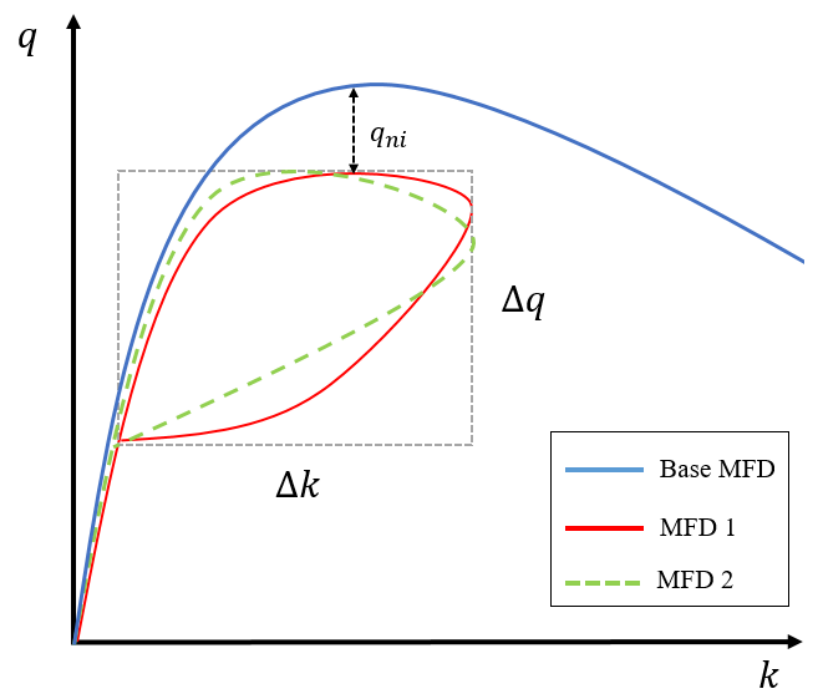

FIGURE 1 : Explanatory variable for hysteresis loops characteristics

TABLE 1 : Explanatory variables to describe MFD with hysteresis loop

\begin{tabular}{|c|c|c|}
\hline Variable & Explanation & Unit \\
\hline$q_{n i}$ & capacity loss & $(\mathrm{veh} / \mathrm{h} / \mathrm{ln})$ \\
\hline$\Delta q$ & flow drop (loop height) & $(\mathrm{veh} / \mathrm{h})$ \\
\hline$\Delta o$ & occupancy changes (loop width) & $(\%)$ \\
\hline$S_{H}$ & area of the loop approximated by rectangular & $(\mathrm{veh} / \mathrm{h})(\%)$ \\
\hline$\overline{v_{H}}$ & average speed over the hysteresis loop & $(\mathrm{km} / \mathrm{h})$ \\
\hline
\end{tabular}




\section{SIMULATION EXPERIMENT}

In this section, we present the framework of a microscopic simulation experiment to investigate our initial postulation and show the results. The discussion on the obtained results is provided in section 5. For the simulation, we use the microscopic traffic simulation SUMO (42). Please note that we are neither aiming at evaluating a realistic scenario nor questioning the equilibrium condition during incidents. The primary goal is to study a controlled environment where drivers route choice behavior remains manageable. Our toy network is represented by a unidirectional grid of size $5 \times 5$ to reduce the problem size. Such a network can represent an urban network with strong directional flows e.g. high demand during morning peak towards the city center. Grid networks were previously used for investigating the relationship between route choice and the shape of the MFD $(24,25,43,44)$. The network is composed of 60 nodes from which 12 (South and West side) are only origins, 12 are only destinations (North and East side) and the 36 in the middle are signalized intersections with $90 \mathrm{~s}$ of cycle length and $40 \mathrm{~s}$ of green time without any offset. The network consists of 60 links with a length of $300 \mathrm{~m}$, each with two lanes and a speed limit of $50 \mathrm{~km} / \mathrm{h}$. We put one loop detector on both lanes of each link. The data aggregation interval is set to 3 minutes. The distance to the stop line is drawn from a uniform distribution for each loop detector. This results in an MFD with little bias as suggested by (43). Additionally, we relax the assumption of complete sensor coverage and investigate the MFDs based on randomly selected $30 \%$ of all detectors. Yet, we observe similar results for this case. We exclude measurements at the generation and destination links as they may cause significant bias in our network variable calculations.

The demand incrementally increases along with origin links as shown in Figure 2, where $q_{\text {in }}$ is additionally incrementally increased over time. The duration of the simulation is 2 hours and 30 minutes, plus 15 minutes in the beginning for warm-up and 15 minutes in the end for egress. For the first hour the demand is increased by a factor between 2 and 8 every 15 minutes and similarly decreases during the second hour. To ensure congestion does not only appear at the boundary edges where inflows are added, we add some random trips in the network with different trip lengths during these two hours to ensure some traffic is cruising in the network as background congestion. We purposefully replicate similar network configuration used in (44) to compare our results with their findings. We compare the MFDs from several scenarios: a base scenario in which we use the demand profile described above and seven different road closure scenarios in each of which a different link is closed from the beginning to the end of the simulation. Drivers are not informed about this incident and those who originally traverse the closed link to reach their destination are only informed about the closure one intersection upstream of the closed road and only then start to look for the fastest alternative. In case there is no alternative available, the closest destination node is assigned to the vehicle as its new destination. This occurs only to vehicles that must drive straight all the way.

\section{Base Scenario}

The base scenario represents a dynamic user equilibrium condition that is achieved by setting the maximum relative standard deviation of travel time for each OD pair to 0.01 as the convergence criterion. The MFD obtained from this scenario serves as our benchmark MFD to which we compare the other MFDs from incident scenarios. Figure 3 shows a small clockwise hysteresis loop during network unloading from 08:00 to 08:45. During this time the standard deviation of occupancy across the links of the network reaches its maximum value. This is a common phenomenon 


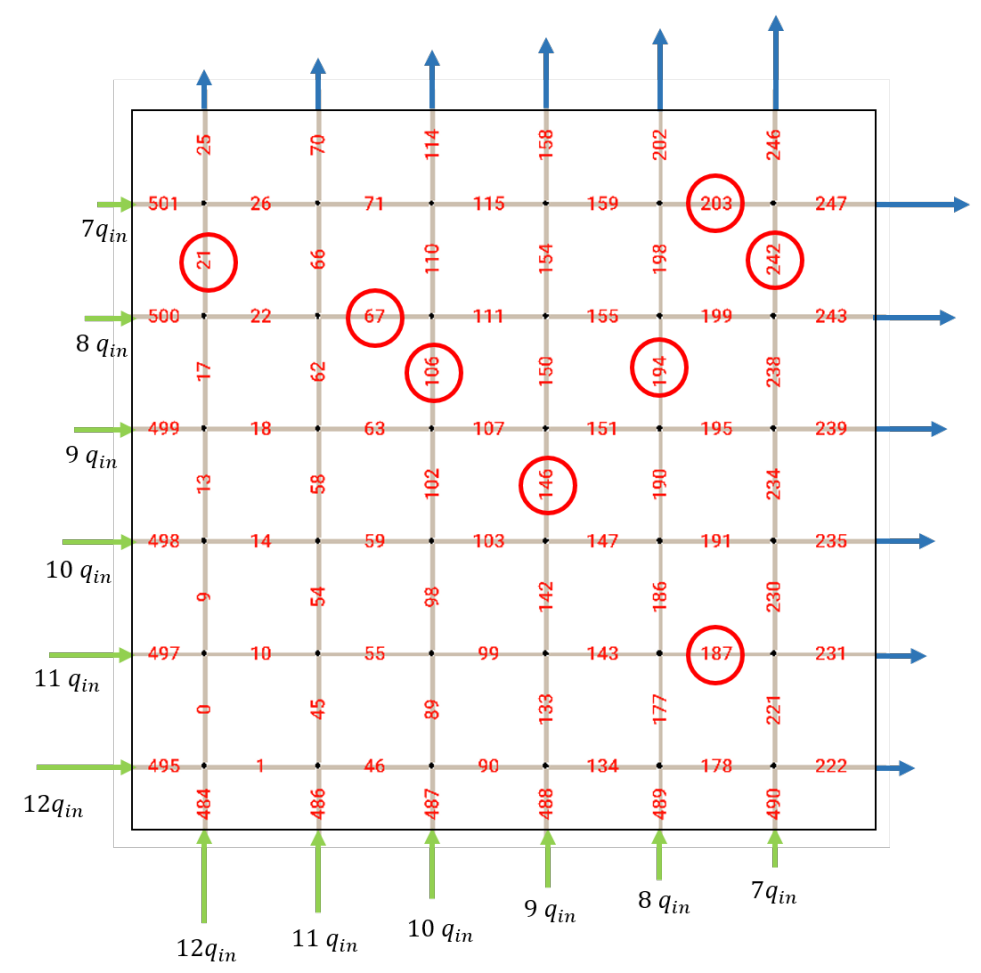

FIGURE 2 : The studied grid-network with the inflow and outflow at its boundaries. Edge IDs with a red circle around their ID are studied for closure scenarios

observed in many other simulation and empirical studies as discussed in Section 2. Please note the smaller loop, right after the hysteresis loop ends, is caused by a sudden increase in network accumulation at 08:45.
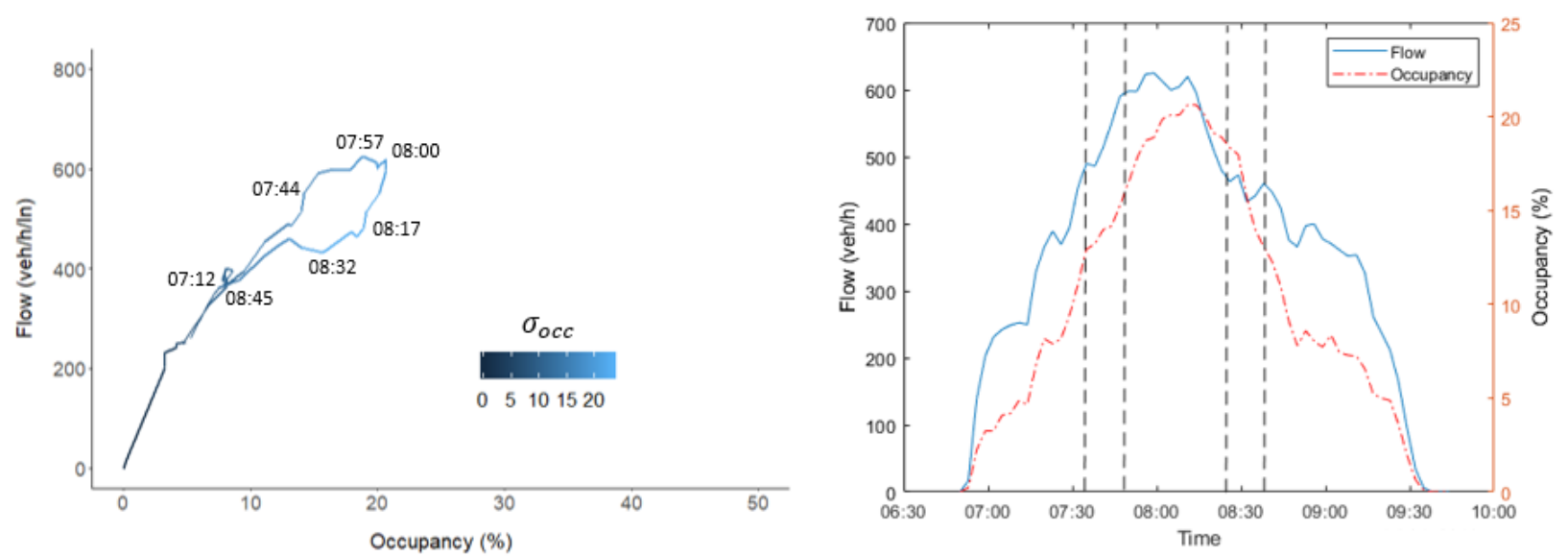

FIGURE 3 : Left: MFD from the base scenario. The standard deviation of occupancy is maximum during unloading part of the hysteresis loop. Right: time series of flow and occupancy showing lower flow for the same occupancy level during unloading. 


\section{Incident Scenarios}

In total, we conduct 8 different incident scenarios by closing links 242, 203, 194, 187, 146, 106, 67 and 21 which can be seen in Figure 2. In order to compare the scenarios, we first calculate the criticality of each link using the introduced CS in section 3. Together with the characteristics of the hysteresis loop obtained from the MFD of each scenario we present an overview of each scenario in Table 2. Generally, the capacity loss $q_{\text {in }}$ decreases linearly as the value of CS decreases. However, there is an exception for the scenario where links 106 and 21 are closed. A possible explanation for this is the strong directional flow in the network from bottom left to the top right. The spillback caused by the closure of these links would extremely limit overall vehicular flow since they are closer to the entry point of demand. In an extreme case, closure of link 21 blocks almost all the possible inflow from the left side of the network resulting in a different demand scenario and thus is not further discussed. Due to the symmetrical topology, links 242 and 203 have identical CS values but due to randomly generated background traffic, the MFDs in these two scenarios are not identical. This can be clearly seen in the capacity drop and the size of the hysteresis loop.

For a better understanding, we compare the links with a similar CS and plot three groups of MFDs as it is shown in the top row of Figure 4. We can see that higher values of CS impose a larger hysteresis loop on the MFD. Moreover, we can easily observe the congested branch of MFD in scenarios 243 and 194. The spillback caused by the closure of these links propagates back to the origins and brings the network close to gridlock. The larger size of the loop implies longer queues and a spillback near to the closed link while other links of the network are in free-flow state. As mentioned above, these observations also hold for the case that only $30 \%$ of all links are equipped with loop detectors.

Another important metric for the analysis of macroscopic traffic dynamics is the trip completion rate which was originally used in (17). It is defined as the number of vehicles exiting the network to the number of vehicles in the network. The decrease in this rate indicates lower network production if the average trip length remains unchanged. The bottom row of Figure 4 depicts the trip completion rate for the three pairs of scenarios. Similar to their MFDs, we observe that the changes in trip completion rates are also very similar in scenarios with close CS values. Please note that this rate is not constant due to changes in average trip length in the network during the loading and unloading phase.

By carefully analyzing the MFDs and the trip completion rate time series, we can observe that in all the scenarios the point at which MFDs during incident situations deviate from the base

TABLE 2 : Criticality score and the characteristics of the hysteresis loop for each scenario

\begin{tabular}{|c|c|c|c|c|c|c|}
\hline Closure scenario & Link CS & $q_{n i}$ & $\Delta q$ & $\Delta o$ & $\mathrm{~S}_{H}$ & $\overline{v_{H}}$ \\
\hline link-242 & 126 & 469 & 262 & 11.4 & 2986 & 31.8 \\
\hline link-203 & 126 & 413 & 206 & 11.1 & 2286 & 31.7 \\
\hline link-194 & 90 & 490 & 202 & 10.2 & 2424 & 34.7 \\
\hline link-146 & 60 & 501 & 122 & 6.14 & 749 & 37.8 \\
\hline link-106 & 40 & 440 & 121 & 4.94 & 597 & 38.0 \\
\hline link-67 & 20 & 542 & 147 & 3.93 & 577 & 38.2 \\
\hline link-187 & 5 & 566 & 118 & 3.37 & 397 & 38.6 \\
\hline link-21 & 1 & 445 & 315 & 6.63 & 2088 & 37.5 \\
\hline
\end{tabular}



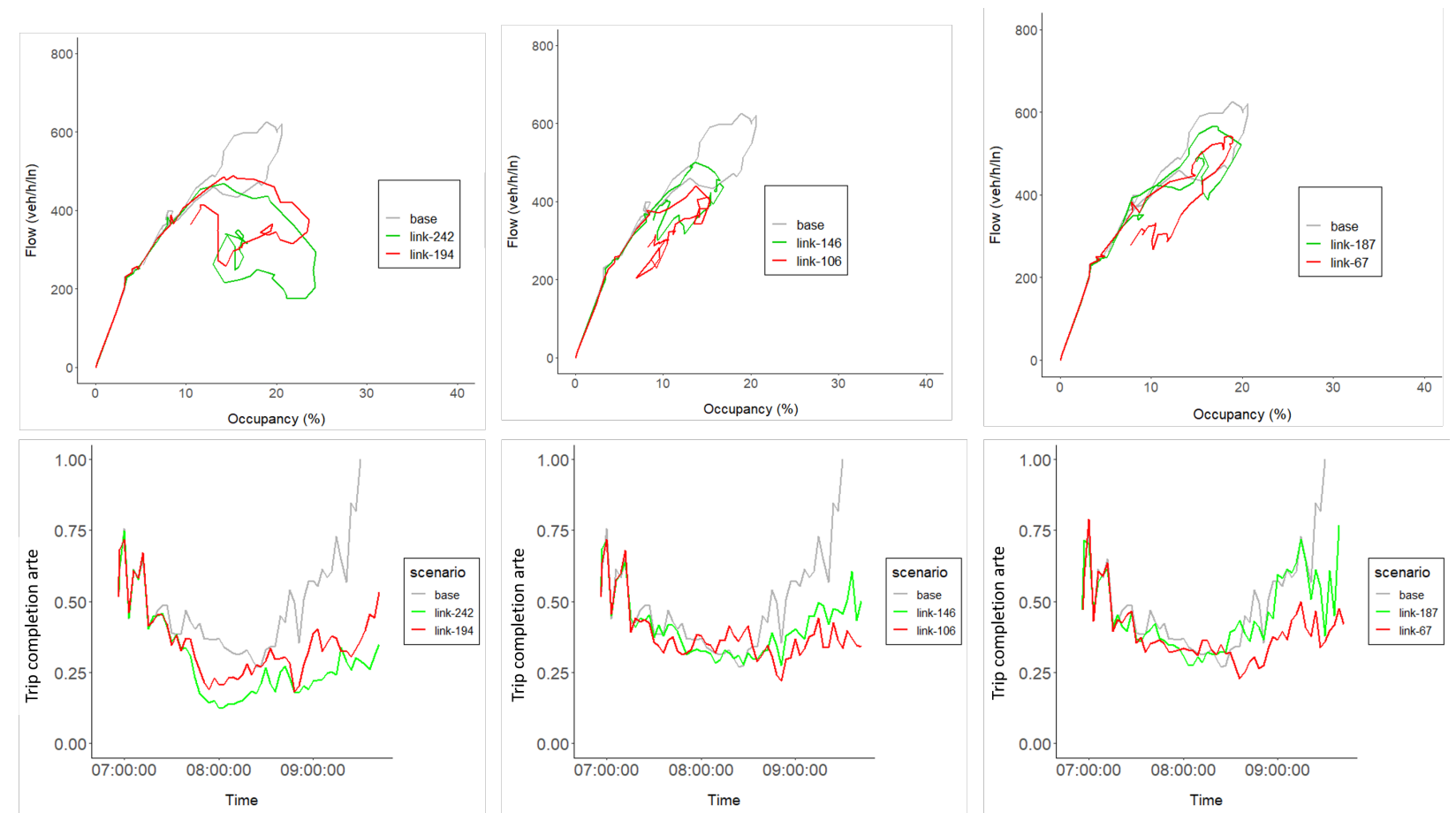

FIGURE 4 : Top: Similar scenarios exhibit comparable hysteresis loops in the MFD. Bottom: trip completion rate for the similar groups of scenarios follow comparable pattern.

scenario MFD is at occupancy of around $8 \%$ and flow of $400 \mathrm{veh} / \mathrm{h} / \mathrm{ln}$. Moreover, the time at which this splitting happens is more or less identical in all of the scenarios. We will discuss the reasons for this in the next section.

Network heterogeneity is a well-studied factor that affects the shape of MFD by inducing scatter and hysteresis loops. Thus, it is necessary to investigate the existence of heterogeneity in more detail. The most common measure to quantify it is the standard deviation of occupancy. The distribution of congestion across the network at different time intervals of the simulation is illustrated in Figure 5. We can see that in some situations some links are fully congested (occupancy $100 \%$ ) and some other empty (occupancy $0 \%$ ). This happens for scenarios in which a link in the middle of the grid network is closed than a link at closer to destination links. By comparing the first and the second row of the histograms, we observe that both mean and standard deviation of occupancy are increasing. Whereas in the third row of histograms, both values decrease again and reach values similar to the ones in the histograms in the first row. Overall, this analysis confirms the distribution of congestion in scenarios 187 and 67 is closer to the base scenario as indicated by the associated MFDs.

\section{DISCUSSION}

After presenting the result of our simulation experiment, we discuss the findings and limitations of our approach. We compare our results to studies that have used the concept of the MFD under other non-recurring congestion and seek potential generalization of the findings.

First of all, we can confirm our initial postulation and conclude that more critical links in the network induce larger hysteresis loop and higher capacity loss in the MFD. These results are in line with those from (39) who have earlier used the concept of the MFD to develop a criticality 

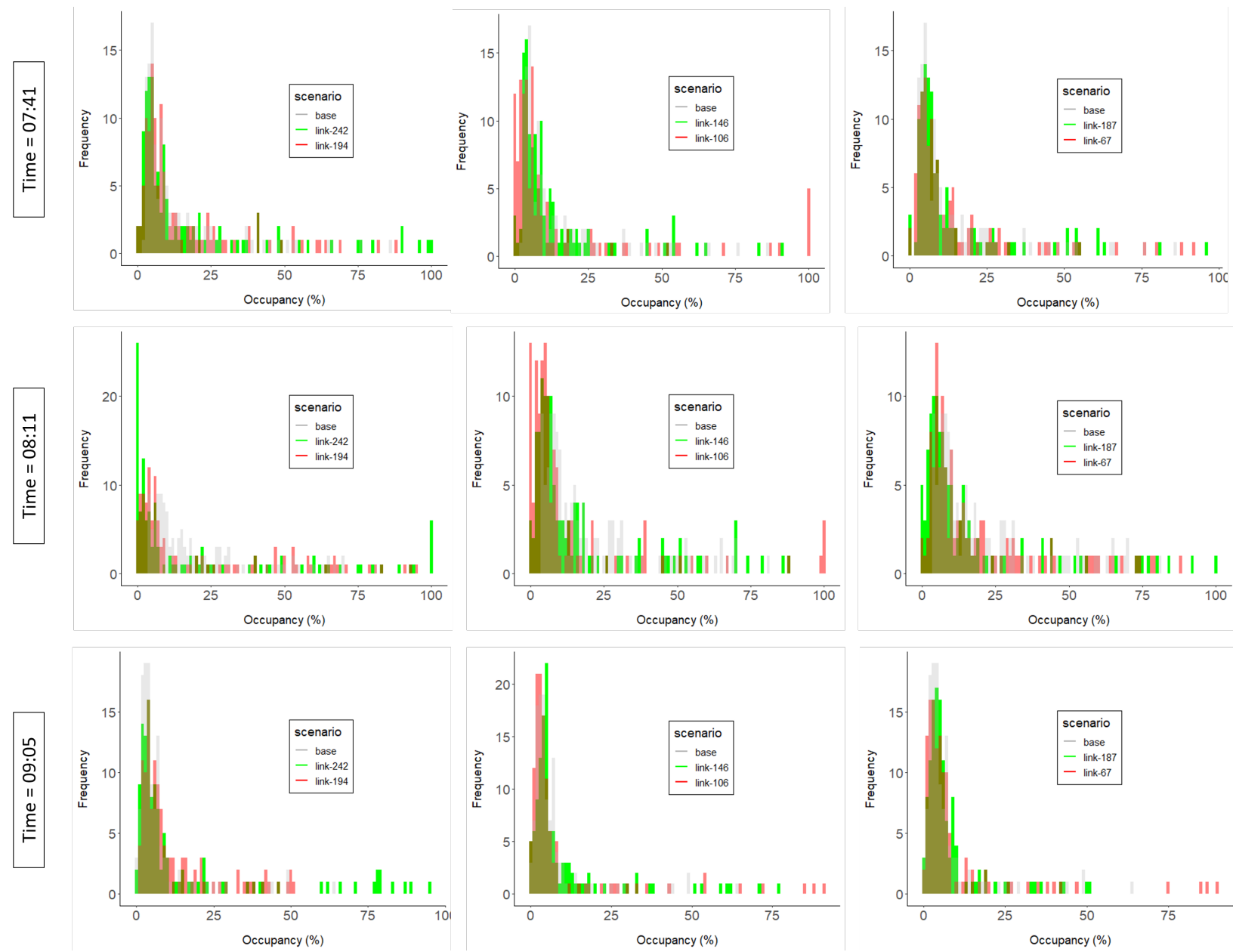

FIGURE 5 : Occupancy distribution across the links of the network during different time intervals for the selected scenarios.

index for links of a network by removing a link from the network and calculating the reduction in weighted average flow. Additionally, we observe that the closure of links with similar CS results in comparable changes in the shape of the MFD. This is particularly interesting since it enables us to develop incident detection algorithms. We can cluster the links of a given network into a limited number of clusters based on their criticality and then detect possible road closures by observing the changes in the shape of MFD. Thus, we can detect an incident by traffic measurements at other locations in the network. Moreover, the proposed CS is a proxy for redundancy in the network which lets us conclude that the MFD in networks with higher redundancy is more stable as drivers have more route choices. Such property was suggested in (37) as well.

As mentioned earlier, we designed our network similar to the one from Parzani et al. (44) to reproduce comparable MFDs. In their paper, the authors divide the network based on a route overlapping factor in five clusters and derive an MFD for each. For the cluster that is mainly composed of links at the center of the network, the MFD shows a higher trip production with a smaller network and therefore, their closure would have minor impacts on the MFD.
. The litical density. This implies that these links contribute less to average 
Another finding of the study is related to the type of hysteresis loops. Two types of hysteresis loops were introduced in (34) We observe a third type of hysteresis loop in which flow increases as occupancy decreases. This can happen during situations where several links of the network are fully congested (at jam density) and inflow is near zero while outflow increases. Imagine a case where a link is saturated and congestion propagates to the origin links which blocks the inflow demand. Consequently, demand remains constant and flow is driven only by supply starts to dissipate. In our specific case, for the scenarios where links 242 and 203 are closed, at the beginning of the simulation the inflow is much larger than the outflow. After the peak in demand is reached, the links of the network are mostly saturated and we have mainly outflow resulting in a significant reduction of the inflow/outflow ratio. Once several links reach free-flow state again, this ratio is balanced and the hysteresis loop is closed. From there, the unloading phase of the network continues on the same curve as the loading phase.

We want to highlight another interesting observation made in this study. The point at which the MFD obtained from the incident scenario diverges from the base MFD was the same in all of scenarios. Interestingly, in the MFD of the base scenario the hysteresis loop starts exactly at the same point. This implies that our network gets unstable at flow $400 \mathrm{veh} / \mathrm{h} / \mathrm{ln}$ and occupancy $8 \%$. This behavior does not change with removing one link from the network for the same demand. Even though we cannot generalize this conclusion, but the existence of a point at which bifurcation occurs as a property of the network is valuable for the development of traffic control measures.

\section{CONCLUSION AND FUTURE WORK}

In this paper, we present a framework to study the macroscopic traffic dynamics in urban networks during incidents. More specifically, we use a microscopic traffic simulation to study the characteristics of the MFD and the induced hysteresis loop due to a road closure. We introduce a link criticality score (CS) which reflects the changes in network redundancy by removing a link from the network. An important finding of the study is that redundancy is directly connected to the shape and size of the hysteresis loop. A higher number of alternative paths alleviates the impacts of road closure by assisting the drivers to find alternative routes which results in an even distribution of congestion across the network. The results of the simulation show that higher CS value imposes larger hysteresis loops in the MFD. Such a property can be used to develop incident detection algorithms for urban networks. The main advantage of this approach is that MFD can be estimated by a limited number of loop detectors which most cities have already in place. In other words, our proposed framework can detect incidents occurring on links without sensors by observing traffic on other links in the same network.

One of the main limitations of this study is that the results may strongly rely on the assumptions regarding the network structure, the OD pairs, and drivers' route choice during incidents. Therefore, future works will focus on different network structures with various demand profiles. Moreover, the behavior of drivers while facing a closed road must be further investigated. The influence of route choice on the shape of MFD suggests that if drivers have access to real-time travel information they may avoid the congestion, which significantly affects the characteristics of the MFD. This was investigated in a previous study by the authors (45) in which they show that implementing rerouting strategies can improve network production and avoid gridlock. Additionally, we assumed detectors are located in a way that bias in the data is minimized. In reality, however, such an assumption is rare and hence the bias from measurement on the shape of the hysteresis loop should be studied. Such bias can also arise from the selection of time-space window for data ag- 
gregation and needs a careful analysis, which was not covered in this study. Finally, our proposed

$2 \mathrm{CS}$ is static and does not consider the traffic condition. This indicator can be further improved by 3 taking into account the traffic volume or travel time of the links.

\section{AUTHORS CONTRIBUTION}

5 The authors confirm contribution to the paper as follows: study conception and design: Sasan 6 Amini and Fritz Busch; Simulation analysis and interpretation of results: Sasan Amini and Gabriel 7 Tilg; draft manuscript preparation: Sasan Amini, Gabriel Tilg and Fritz Busch. All authors re8 viewed the results and approved the final version of the manuscript. 


\section{REFERENCES}

1. Busch, F., Incident detection. In Concise Encyclopedia of Traffic \& Transportation Systems, Elsevier, 1991, pp. 219-225.

2. Mahmassani, H., C. Haas, S. Zhou, and J. Peterman, Evaluation of incident detection methodologies. Research Report No. FHWA/TX-00/1795-1, 1999.

3. Persaud, B. N., F. L. Hall, and L. M. Hall, Congestion identification aspects of the McMaster incident detection algorithm. Transportation Research Record, , No. 1287, 1990.

4. Margreiter, M., Automatic incident detection based on bluetooth detection in northern Bavaria. Transportation research procedia, Vol. 15, 2016, pp. 525-536.

5. Busch, F. and M. Fellendorf, Automatic incident detection on motorways. Traffic Engineering \& Control, Vol. 31, No. 4, 1990.

6. Bell, M. G. and B. Thancanamootoo, Automatic incident detection within urban traffic control systems. Proceedings of the Roads and Traffic Conference, Vol. 4, No. 2, 1988.

7. Sethi, V., N. Bhandari, F. S. Koppelman, and J. L. Schofer, Arterial incident detection using fixed detector and probe vehicle data. Transportation Research Part C: Emerging Technologies, Vol. 3, No. 2, 1995, pp. 99-112.

8. Yuan, F. and R. L. Cheu, Incident detection using support vector machines. Transportation Research Part C: Emerging Technologies, Vol. 11, No. 3-4, 2003, pp. 309-328.

9. Anbaroglu, B., B. Heydecker, and T. Cheng, Spatio-temporal clustering for non-recurrent traffic congestion detection on urban road networks. Transportation Research Part C: Emerging Technologies, Vol. 48, 2014, pp. 47-65.

10. Anbaroğlu, B., T. Cheng, and B. Heydecker, Non-recurrent traffic congestion detection on heterogeneous urban road networks. Transportmetrica A: Transport Science, Vol. 11, No. 9, 2015, pp. 754-771.

11. Zhang, Z., Q. He, H. Tong, J. Gou, and X. Li, Spatial-temporal traffic flow pattern identification and anomaly detection with dictionary-based compression theory in a large-scale urban network. Transportation Research Part C: Emerging Technologies, Vol. 71, 2016, pp. 284302.

12. Godfrey, J., The mechanism of a road network. Traffic Engineering \& Control, Vol. 8, No. 8, 1969.

13. Ardekani, S. and R. Herman, Urban Network-Wide Traffic Variables and Their Relations. Transportation Science, Vol. 21, No. 1, 1987, pp. 1-16.

14. Herman, R. and I. Prigogine, A two-fluid approach to town traffic. Science, Vol. 204, No. 4389, 1979, pp. 148-151.

15. Mahmassani, H., J. C. Williams, and R. Herman, Performance of urban traffic networks. In Proceedings of the 10th International Symposium on Transportation and Traffic Theory, 1987, pp. 1-20.

16. Daganzo, C. F. and N. Geroliminis, An analytical approximation for the macroscopic fundamental diagram of urban traffic. Transportation Research Part B: Methodological, , No. 42, 2008, pp. 771-781.

17. Geroliminis, N. and C. F. Daganzo, Existence of urban-scale macroscopic fundamental diagrams: Some experimental findings. Transportation Research Part B: Methodological, Vol. 42, No. 9, 2008, pp. 759-770.

18. Buisson, C. and C. Ladier, Exploring the impact of homogeneity of traffic measurements on the existence of macroscopic fundamental diagrams. Transportation Research Record: Journal of 
the Transportation Research Board, Vol. 2124, 2009, pp. 127-136.

19. Tsubota, T., A. Bhaskar, and E. Chung, Macroscopic fundamental diagram for Brisbane, Australia. Transportation Research Record: Journal of the Transportation Research Board, Vol. 2421, 2014, pp. 12-21.

20. Ambühl, L., A. Loder, M. Menendez, and K. W. Axhausen, Empirical macroscopic fundamental diagrams: New insights from loop detector and floating car data. Transportation Research Record, 2016.

21. Ambühl, L., A. Loder, H. Becker, M. Menendez, and K. W. Axhausen, Evaluating London's congestion charge: An approach using the macroscopic fundamental diagram. In 7th Transport Research Arena (TRA 2018), IVT, ETH Zurich, 2018.

22. Ji, Y., W. Daamen, S. Hoogendoorn, S. Hoogendoorn-Lanser, and X. Qian, Investigating the Shape of the Macroscopic Fundamental Diagram Using Simulation Data. Transportation Research Record: Journal of the Transportation Research Board, Vol. 2161, No. 1, 2010, pp. 40-48.

23. Knoop, V. L., D. De Jong, and S. P. Hoogendoom, Influence of road layout on network fundamental diagram. Transportation Research Record, Vol. 2421, No. 1, 2014, pp. 22-30.

24. Duruisseau, C. and L. Leclercq, A Global Sensitivity Analysis of Dynamic Loading and Route Selection Parameters on Network Performances. Journal of Advanced Transportation, Vol. 2018, 2018.

25. Knoop, V., S. Hoogendoorn, and J. Van Lint, Routing Strategies Based on Macroscopic Fundamental Diagram. Transportation Research Record: Journal of the Transportation Research Board, Vol. 2315, No. 2315, 2013, pp. 1-10.

26. Yildirimoglu, M., M. Ramezani, and N. Geroliminis, Equilibrium analysis and route guidance in large-scale networks with MFD dynamics. Transportation Research Procedia, Vol. 9, 2015, pp. 185-204.

27. Yildirimoglu, M., I. I. Sirmatel, and N. Geroliminis, Hierarchical control of heterogeneous large-scale urban road networks via path assignment and regional route guidance. Transportation Research Part B: Methodological, Vol. 118, 2018, pp. 106-123.

28. Haddad, J., Robust constrained control of uncertain macroscopic fundamental diagram networks. Transportation Research Procedia, Vol. 7, 2015, pp. 669-688.

29. Keyvan-Ekbatani, M., A. Kouvelas, I. Papamichail, and M. Papageorgiou, Exploiting the fundamental diagram of urban networks for feedback-based gating. Transportation Research Part B: Methodological, Vol. 46, No. 10, 2012, pp. 1393-1403.

30. Cao, J. and M. Menendez, System dynamics of urban traffic based on its parking-related-states. Transportation Research Part B: Methodological, Vol. 81, 2015, pp. 718-736.

31. Leclercq, L., A. Sénécat, and G. Mariotte, Dynamic macroscopic simulation of on-street parking search: A trip-based approach. Transportation Research Part B: Methodological, Vol. 101, 2017, pp. 268-282.

32. Gayah, V. and C. F. Daganzo, Clockwise hysteresis loops in the Macroscopic Fundamental Diagram: An effect of network instability. Transportation Research Part B: Methodological, Vol. 45, No. 4, 2011, pp. 643-655.

33. Saberi, M., H. S. Mahmassani, and A. Zockaie, Network capacity, traffic instability, and adaptive driving: findings from simulated urban network experiments. EURO Journal on Transportation and Logistics, Vol. 3, No. 3-4, 2014, pp. 289-308.

34. Saberi, M. and H. S. Mahmassani, Hysteresis and Capacity Drop Phenomena in Freeway Net- 
works Empirical Characterization and Interpretation. Transportation Research Record: Journal of the Transportation Research Board, Vol. 2391, 2013, pp. 44-55.

35. Geroliminis, N. and J. Sun, Properties of a well-defined macroscopic fundamental diagram for urban traffic. Transportation Research Part B: Methodological, Vol. 45, No. 3, 2011, pp. 605-617.

36. Shim, J., J. Yeo, S. Lee, S. H. Hamdar, and K. Jang, Empirical evaluation of influential factors on bifurcation in macroscopic fundamental diagrams. Transportation Research Part C: Emerging Technologies, Vol. 102, 2019, pp. 509-520.

37. Geroliminis, N. and J. Sun, Hysteresis phenomena of a Macroscopic Fundamental Diagram in freeway networks. Transportation Research Part A: Policy and Practice, Vol. 45, No. 9, 2011, pp. 966-979.

38. Zockaie, A., H. S. Mahmassani, M. Saberi, and Ö. Verbas, Dynamics of Urban Network Traffic flow during a Large-Scale Evacuation. Transportation Research Record: Journal of the Transportation Research Board, Vol. 2422, No. 1, 2014, pp. 21-33.

39. Kim, S. and H. Yeo, Evaluating Link Criticality of Road Network based on the Concept of Macroscopic Fundamental Diagram Diagram. Transportmetrica A: Transport Science, Vol. 13, No. 2, 2016, pp. 162-193.

40. Horiguchi, R., M. Iijima, M. Kobayashi, and H. Hanabusa, Traffic Anomaly Detection for Surface Street Networks With the Mesh-Wised Traffic Indices on Macroscopic Fundamental Diagram. OPTIMUM 2013 - International Symposium on Recent Advances in Transport Modelling, 2013, pp. 1-7.

41. White, D. R. and S. P. Borgatti, Betweenness centrality measures for directed graphs. Social networks, Vol. 16, No. 4, 1994, pp. 335-346.

42. Lopez, P. A., M. Behrisch, L. Bieker-Walz, J. Erdmann, Y.-P. Flötteröd, R. Hilbrich, L. Lücken, J. Rummel, P. Wagner, and E. Wießner, Microscopic Traffic Simulation using SUMO. In The 21st IEEE International Conference on Intelligent Transportation Systems, IEEE, 2018.

43. Leclercq, L., N. Chiabaut, and B. Trinquier, Macroscopic Fundamental Diagrams: A crosscomparison of estimation methods. Transportation Research Part B: Methodological, Vol. 62, 2014, pp. 1-12.

44. Parzani, C., L. Leclercq, N. Benoumechiara, and D. Villegas, Clustering route choices methodology for network performance analysis. Transportmetrica B: Transport Dynamics, Vol. 5, No. 2, 2017, pp. 191-210.

45. Amini, S., G. Tilg, and F. Busch, Evaluating the impact of real-time traffic control measures on the resilience of urban road networks. In 2018 21st International Conference on Intelligent Transportation Systems (ITSC), IEEE, 2018, pp. 519-524. 